\title{
Traduire
}

Une autre perspective sur r tr traduction

Revue française de la traduction

$235 \mid 2016$

Luxe, mode... et traduction !

\section{Langage du luxe, luxe du langage}

\section{Laura Cattaneo}

Traducteur : Christine Cross

\section{(2) OpenEdition}

Journals

Édition électronique

URL : https://journals.openedition.org/traduire/829

DOI : 10.4000/traduire.829

ISSN : 2272-9992

Éditeur

Société française des traducteurs

Édition imprimée

Date de publication : 1 décembre 2016

Pagination : 5-11

ISSN : 0395-773X

Référence électronique

Laura Cattaneo, «Langage du luxe, luxe du langage », Traduire [En ligne], 235 | 2016, mis en ligne le 01 décembre 2018, consulté le 05 juillet 2021. URL : http://journals.openedition.org/traduire/829 ; DOI : https://doi.org/10.4000/traduire.829 


\section{Langage du luxe, luxe du langage}

\section{Laura Cattaneo}

L'objet de cet article est de partager quelques propos sur le langage du luxe, en me référant essentiellement à la langue italienne, et ce du point de vue d'une professionnelle des métiers linguistiques.

Penchons-nous tout d'abord sur le mot " luxe " lui-même. De nos jours, le terme "luxe " est tellement galvaudé qu'on peut l'appliquer à presque tout et n'importe quoi, du papier hygiénique à la joaillerie. II en résulte que les marques, produits et services véritablement haut de gamme l'ont plus ou moins banni de leur vocabulaire, en le remplaçant - dans le meilleur des cas - par un registre verbal destiné à transmettre au consommateur la notion de luxe intrinsèque à chaque marque, passant ainsi du " luxe " à "l'expérience du luxe ". En italien, le luxe se dit lusso. Tout comme l'adjectif correspondant lussuoso et des dérivés tels que deluxe et prestigioso, il a connu le même sort que son équivalent dans d'autres langues : à force d'être utilisé à tort et à travers, le mot a perdu son sens d'origine, son caractère élitiste.

L'une des stratégies pour compenser l'utilisation excessive de ces termes consiste à les remplacer par d'autres, moins surfaits, capables de communiquer les sensations, l'expérience même du luxe. Dans le secteur de l'automobile par exemple, une grande marque haut de gamme britannique qui figure parmi mes clients a donné la consigne à tous ses traducteurs des langues de l'UE de remplacer systématiquement le mot luxury qu'ils pourraient retrouver dans le texte original anglais (le mot n'a donc pas encore tout à fait disparu de la langue anglaise) par celui de premium. Mais, en réalité, la question se pose : ces deux termes sontils vraiment interchangeables? Pour moi, premium renferme la promesse de prestations ou de produits complémentaires ou de qualité supérieure en échange d'un prix plus élevé, tandis que luxury est une notion plus générale, plus globale, un idéal à viser indépendamment de la dimension pécuniaire. La marque semble, pourtant, considérer le marché cible incapable de saisir cette nuance, et juge que premium décrit parfaitement bien le concept de luxury ou que les deux vocables sont synonymes. 
Une autre stratégie, bien plus structurée que celle que nous venons d'évoquer, vise à susciter le sentiment du luxe sans jamais en prononcer le nom. II s'agit dans ce cas de créer un langage ad hoc spécifique à la marque qui symbolise ses valeurs, son identité et son caractère exclusif, incarnant ainsi sa conception du luxe. Tout cela constitue un défi intéressant pour le traducteur ou le transcréateur, qui devra suivre le même cheminement intellectuel que les auteurs pour arriver au même résultat dans la langue cible, tout en allant encore plus loin. II ne s'agit pas de faire passer le langage du luxe d'une langue à l'autre mais de recréer intégralement l'univers de la marque dans la langue cible en reproduisant fidèlement ses caractéristiques intrinsèques et en assurant une bonne adéquation à la culture et aux sensibilités du public cible.

Par leurs choix langagiers, les marques de luxe ont le potentiel de se distinguer de la concurrence. Le langage se doit d'être châtié et le registre verbal choisi avec soin pour incarner aussi bien l'identité de la marque que celle de sa clientèle. Le secteur hôtelier, un de mes domaines de prédilection, regorge d'exemples de cette diversité, que l'on peut trouver par exemple dans un seul et même segment, celui du luxe.

Celles et ceux qui ont eu l'occasion de parcourir les descriptions, souvent d'une banalité affligeante, des hôtels multi-étoilés savent qu'ils disposent toujours de chambres luxueuses, de prestations haut de gamme, de suites prestigieuses, de programmes de fidélité réservés à l'élite, etc. Traduites en italien, ces descriptions sont tout aussi banales, croyez-moi. Par quels moyens un hôtel ou une chaîne d'hôtels de luxe peut-il se démarquer du lot ? Le langage et la communication sont des outils formidables à cet égard. Citons l'exemple d'un grand groupe d'hôtels mondialement connu.

Ce groupe est propriétaire de marques diverses et variées, certaines figurant dans le palmarès de tête des hôtels haut de gamme. Juste avant la grande crise de 2008, à l'époque où de nombreuses entreprises se permettaient encore d'investir lourdement dans le langage et la communication, le groupe en question a réussi un tour de force en cernant l'essence même de chacune de ses marques pour mieux lui attribuer une identité verbale et un registre spécifique. Chaque marque décrivait ses prestations, son offre commerciale et ses promotions d'une voix particulière, reprise par ses collaborateurs mais aussi par les supports physiques distribués dans les chambres.

J'ai eu la chance de participer au processus d'adaptation du style de communication américain à son équivalent italien et je garde en mémoire le souvenir de tous les échanges très constructifs que j'ai pu avoir avec le personnel et la direction des hôtels pour décider de la manière de reprendre le concept et de le moduler en fonction du marché italien, en diluant le côté trop " américain " et en évitant les erreurs dans la localisation des piliers de la marque.

La première série d'hôtels du groupe a, pour argument de vente, le bien-être et le rajeunissement. Elle met l'accent sur le " bien manger " (aliments nutraceutiques) à grand renfort 
d'expressions comme coccolarsi (se dorloter), benessere (le bien-être), massaggi (les massages), sprofondare (s'enfoncer - sous-entendu dans un lit ultraconfortable), energia (l'énergie), energizzante (énergisant), sano (sain), mangiare bene (bien manger), nutriente (nourrissant), ricaricarsi (recharger ses batteries), vitalità (la vitalité) : somme toute, un langage choisi pour suggérer le confort et le bien-être dans lesquels la clientèle pourra se prélasser. Alors que le discours américain d'origine s'orientait davantage vers la notion d'énergie, le choix pour les antennes italiennes s'est plutôt porté sur le côté " cocooning " de la marque. À titre d'exemple, aux États-Unis les chambres étaient équipées d'un lit dit " céleste " conçu pour donner au client la sensation de s'immerger dans un confort absolu, tandis que les hôtels italiens ont poussé le concept plus loin en proposant une carte " oreiller céleste ", très prisée des clients, et en déclinant cette offre à tous les niveaux, y compris dans la communication et les supports écrits.

Vient ensuite une autre chaîne d'hôtels ayant pour thème l'art, la culture, la mode et la cuisine gastronomique. Le langage employé par cette marque est riche en tradizioni artistiche (traditions artistiques), dettagli di design (détails conceptuels), tonalità del legno (nuances de bois), esplorare (exploration), ispirazione (inspiration), cultura del caffè (passion du café), sfizioso e raffinato (goûteux et raffiné). Un monde qui se décrit comme étant le luogo d'incontro delle menti creative (lieu de rencontre des esprits créateurs) où, par affinité, l'on se sent chez soi. L'Europe, et l'Italie en particulier, ont accentué le côté artistique en mettant en place pour chaque lieu d'implantation un programme d'événements culturels dont la clientèle pourra profiter pendant son séjour. De surcroît, l'antenne italienne a travaillé avec un célèbre torréfacteur italien pour créer une "carte des cafés " exportée ensuite par la marque à travers le monde.

La particularité de la troisième marque hôtelière du groupe est son côté tendance, branché et " jeunesse d'aujourd'hui ». Le langage adopté est donc truffé de fantastico (fantastique), arredi vivaci (mobilier exubérant), accessori originali (accessoires uniques), letti esclusivi (lits exclusifs) et un'esperienza intrigante, fresca e divertente (expérience intrigante, rafraîchissante et divertissante). Tout est exagéré, y compris le langage. Mais si aux États-Unis on appelle les clients par leur prénom, en Europe, surtout dans les pays latins, il a fallu adopter une autre démarche pour tenir compte des différences culturelles et éviter d'offusquer les visiteurs.

La dernière marque du groupe constitue le nec plus ultra du secteur hôtelier : un endroit renommé pour son caractère exclusif. Le langage de cette marque fourmille de esperienze indimenticabili (expériences inoubliables), senza paragoni (sans pareil), spettacolare (spectaculaire), raffinatezza (sophistication), sontuoso (somptuosité), affascinante (fascinant) et autres impeccabile (impeccables) : un vocabulaire un tantinet excessif et un peu trop quelconque pour une marque de cet acabit. Dans ce cas, malheureusement, l'équipe italienne n'a pas eu son mot à dire dans le choix du registre à adopter au plan local, d'où des formules parfois lourdes pour une oreille italienne. 
Les quatre chaînes hôtelières, toutes plutôt haut de gamme, ont ainsi réussi à conserver leur originalité et à transmettre une notion et une expérience de luxe différentes dans chaque cas, ne serait-ce que grâce aux différences de registre et de langue qui font écho à la diversité des décors, des normes, des ambitions et de clientèle. L'identité visuelle est certes un élément incontournable dans la création d'une image de marque, mais le choix du langage ajoute un volet supplémentaire en permettant au public d'imaginer et d'anticiper l'expérience à venir, faisant ainsi résonner la marque.

Au vu de ces exemples, on pourrait dire que le luxe est le langage des émotions car, comme nous l'avons déjà dit, on est passé de la simple mention du mot "luxe "à une explication des raisons présidant à la catégorisation de telle ou telle chose dans l'univers du luxe. Toutes ces marques communiquent leur idée du luxe par un choix de vocabulaire symbolisant leur positionnement unique sur le marché. II semblerait donc que le langage du luxe se décline au pluriel, au moins aujourd'hui, chacune de ses variantes servant à relier le positionnement de la marque, voire à conforter son authenticité. C'est presque comme si chaque marque avait ciblé un aspect différent du luxe. Dans l'idéal, chaque marque haut de gamme devrait avoir une écriture qui lui est propre, ce qui est très difficile à reproduire dans d'autres langues. De ce fait, la présence de professionnels de la traduction et de la transcréation est absolument essentielle dans les campagnes multinationales.

Traduire des mots est chose facile - des mots tels que " luxe ", " élégance " " prestige ", mais le sens qui se cache derrière ces mots peut varier en fonction du pays et de la culture, une langue étant nourrie par ceux qui la parlent et l'écrivent, leur mentalité, leur sensibilité et, de nos jours, les technologies qu'ils utilisent. L'écriture, et à plus forte raison, la traduction et la transcréation, sont des tâches à caractère artisanal. Façonner un langage ad hoc et un univers de la communication autour d'une marque est un luxe en soi, tout comme le travail fait à la main qui est devenu un élément de plus en plus central de notre perception actuelle du luxe véritable.

Dans sa composition, le luxe contemporain est très lié au concept de personnalisation. Toujours dans le secteur hôtelier, ce concept a pris une telle importance ces dernières années qu'il a même pu enfanter la notion de " collections " d'hôtels : autrement dit, de groupes hôteliers où chaque hôtel dispose de ses propres concepts, de sa propre ambiance, de ses propres rythmes, de sa propre identité visuelle et de ses propres normes. II s'agit plutôt de regroupements d'hôtels de luxe indépendants, ayant chacun leur personnalité tout en respectant les mêmes principes en matière d'accueil et en ayant recours aux mêmes filières de commercialisation. Ils doivent leur charme et leur réputation à l'accueil unique et individuel qu'ils réservent à leurs clients. Sur le plan linguistique, cette personnalisation représente un défi supplémentaire que doivent relever les auteurs et les transcréateurs, car il leur faut créer et mettre en contexte un langage et un registre verbal uniques, non plus pour une marque dans son ensemble mais pour chaque hôtel pris individuellement, en tenant compte de ses atouts uniques, de sa personnalité et de ses prestations spécifiques. 
Composition. Personnalisation. Relativité. Comme l'a constaté à juste titre l'université italienne de Sassari dans une étude sur les voyages haut de gamme, le luxe est quelque chose de subjectif (laissé à l'appréciation de chacun), de dynamique (évoluant dans le temps) et de relatif (tributaire du contexte culturel).

Le concept de luxe change et évolue dans le temps : lorsque mes parents se sont mariés en 1960, posséder un téléviseur constituait un véritable luxe. Aujourd'hui, tout le monde en a un, hormis ceux qui considèrent que le vrai luxe est de chasser cet objet de leur vie. Mais dans les pays en voie de développement, ce constat ne tient pas. II va sans dire que le langage, en tant que témoin de la vie, évolue mais dans des proportions sans doute moins importantes. À moins que ce ne soit pas tant la langue qui évolue que la manière dont on l'utilise. Le transcréateur doit donc peser tous ces facteurs dans la balance et devient, du coup, expert en curation de contenus, ce qui n'est pas forcément une tâche facile.

Prenons le cas des campagnes internationales de promotion conçues aux États-Unis devant être transposées dans un contexte européen, où le concept de luxe peut prendre une tout autre allure. Un exemple plutôt cocasse à ce sujet concerne l'univers de la mode, où le langage adopté par les marques italiennes, y compris celles qui appartiennent à des entreprises italiennes, est de plus en plus souvent façonné en anglais avant d'être transposé en italien. Les campagnes promotionnelles internationales sont souvent confiées à de grosses entreprises de communication et de promotion basées aux États-Unis ou en Grande-Bretagne, qui préfèrent élaborer leur campagne en anglais puis l'adapter, le cas échéant, aux autres marchés. L'expression " le cas échéant " ne s'est pas glissée par hasard dans cette phrase, car il peut s'avérer parfois plus simple - autrement dit plus avantageux financièrement - de tout laisser en anglais.

Ainsi, les transcréateurs de langue italienne peuvent se retrouver à adapter la campagne de promotion d'une marque italienne de mode inspirée d'un concept italien mais façonnée et finalisée en anglais avant d'être (re)localisée en italien. Je pense plus particulièrement à une campagne de cette nature que le client a dû adapter au marché italien - contrairement aux autres marchés -, la version anglaise étant susceptible de laisser le public italien complètement pantois.

Me vient à l'esprit également le cas d'une autre campagne importante, cette fois-ci pour le compte d'une marque de produits de verrerie appartenant désormais à une maison italienne, qui a commencé sa vie en langue anglaise avant de passer entre les mains d'une équipe de transcréateurs européens. Cette campagne a nécessité un gros travail d'adaptation, car la version originale était truffée de références culturelles spécifiques au monde anglo-saxon, à mille lieues de l'univers des langues néolatines. Le client a pourtant décidé au final d'imposer la version anglaise à peu près partout... D'énormes affiches sont ainsi venues habiller les murs des villes contenant des jeux de mots en anglais totalement hermétiques, non pas en raison 
d'une mauvaise traduction mais du fait de l'absence de traduction. La marque a beau être haut de gamme, ses sensibilités en matière de communication ont manqué cette fois-là de finesse, ne serait-ce que d'un point de vue linguistique.

Toutefois, les agences les plus intelligentes impliquent des transcréateurs du pays cible dès le tout début d'une campagne et procèdent à des essais sur les différents marchés nationaux avant de boucler leur démarche. En travaillant ainsi, on arrive à un concept dont la localisation s'avérera relativement facile, tout en minimisant le risque d'un résultat bancal ou, pire encore, d'un échec monumental.

Si l'Italie est généralement reconnue pour sa culture, son Art avec un grand " A ", son excellence culinaire et sa mode, ce potentiel énorme n'est pas toujours bien exploité. Le choix du bon langage et du bon registre dans la création d'une ambiance de luxe peut contribuer à l'amélioration de l'image du pays au plan international et faciliter les efforts de marketing des marques et des produits italiens haut de gamme. Le langage peut aider à raconter et à façonner leur histoire.

Dans le domaine du luxe, le langage lui-même peut être vecteur de luxe. Se lancer dans la création d'un langage capable de véhiculer sa propre conception du luxe, de la décrire pour permettre à l'autre de la ressentir, de la vivre comme s'il y était, n'est-ce pas un luxe en soi ? En tant que professionnelle des langues et de la communication, je ne vois vraiment pas l'intérêt de préciser dans le détail les caractéristiques de votre marque, son originalité et ses spécificités et d'aller jusqu'à choisir des images et des supports éloquents et accrocheurs, si ensuite votre communication n'est qu'un enchaînement de qualificatifs quelconques, comme " personnalisé " ou " exclusif ". Lorsqu'une marque vante ses atouts ainsi, les mots finissent par perdre tout leur sens. Les contenus et l'identité de la marque également. Pour véritablement se démarquer, il faut créer une identité verbale qui raconte l'histoire de la marque, en évitant le simple récit et en s'inspirant de faits réels : I'histoire et les aspirations des collaborateurs, les valeurs qui les habitent, la manière dont ils les expriment et les utilisent pour donner corps aux prestations et aux produits de la marque, à sa conception unique de l'univers du luxe. Langage du luxe, luxe du langage.

info@lauracattaneo.eu

Traduit de l'anglais par Christine Cross 
De langue maternelle italienne, Laura Cattaneo est une professionnelle chevronnée de la traduction, de la transcréation, de la révision et de la curation de contenus, dont les langues de travail sont l'anglais et le français. Ses domaines de prédilection sont, d'une part, la traduction et la transcréation de textes de marketing et la publicité. Après des études de langues, elle s'est lancée dans le marketing et la gestion des créations au service du secteur hôtelier, un apprentissage au travail en international, à la communication d'entreprise et aux nuances multiples de l'univers du luxe qui lui a permis de se munir d'atouts désormais exploités dans son rôle de traductrice freelance axée sur le langage et la communication. En tant que professionnelle indépendante travaillant pour son compte, Laura conseille également les entreprises, qu'il s'agisse de multinationales ou ciblant des secteurs de niche ou du charme, pour les aider à adapter leur approche du marché italien aux attentes du public local.

Membre accrédité d'ANITI (Association nationale italienne de traducteurs et d'interprètes) Membre accrédité (MCIL) de CiOL (Chartered Institute of linguists, UK)

\section{Bibliographie}

HOSPITALITY NEWS, II turismo del lusso, http://www.hospitality-news.it/lusso/1459-il-turismodel-lusso-dalla-ricerca-universitaria-di-giacomo-del-chiappa.html, consulté en juillet 2016.

BERRY Christopher J., 1994, The Idea of Luxury: A Conceptual and Historical Investigation, Cambridge University Press.

HEARST MAGAZINES UK, The New Language of Luxury: How Millennials are changing the shape of luxury, http://www.hearst.co.uk/news/new-language-luxury-millennials-changing-shape-luxury, consulté en juillet 2016.

RICCA Manfredi et ROBINS Rebecca, 2012, Meta-Luxury, Brands and the Culture of Excellence, Palgrave MacMillan.

DE BEER Megan, "The Language of Luxury ", Tiny Hunter, http://tinyhunter.com.au/blog/ language-luxury, consulté en juillet 2016.

WHITE WILSON Sarah, The Language of Luxury: Content, Translation, \& Culture In Between, http://luxurysociety.com/articles/2012/11/the-language-of-luxury-content-translation-culturein-between, consulté en juillet 2016. 